\title{
Food additive emulsifier restriction is feasible in people with Crohn's disease
}

Crohn's disease is increasing in prevalence globally and this within-generation increase implicates lifestyle factors in disease aetiology. There is a positive association between a 'Western' diet, which is high in food-additive emulsifiers, and the prevalence of Crohn's disease. Meanwhile in vitro and murine studies demonstrate mechanisms through which emulsifiers modify the microbiome, encourage microbial encroachment of the gut epithelium, increase gut permeability and exacerbate colitis ${ }^{1}$. However, emulsifiers are ubiquitous in diet and it is unknown whether a low emulsifier diet is feasible and nutritionally replete. The aim of this study was to assess whether dietary restriction of emulsifiers is feasible in people with Crohn's disease.

Adherence, feasibility and acceptability to a 14-day low emulsifier diet was assessed in 20 participants with stable Crohn's disease. Participants were recruited using promotion through a patient charity. The intervention was a 14-day low emulsifier diet designed to exclude all food-additives classified as emulsifiers (66 in total, based on the Joint FAO/WHO Expert Committee on Food Additives and the Codex Alimentarius classifications of emulsifiers). The low emulsifier diet was delivered by dietetic counselling, an educational booklet and a novel smartphone application. Crohn's disease-related symptoms (patient-reported outcome-2), perceived disease control (IBD-C-8 questionnaire), anthropometry (bioelectrical impedance analysis), nutrient intake (7-day food diary) and food-related quality of life (FR-QoL) were measured at baseline and at the end of the 14-days of low emulsifier diet. Continuous data were compared between baseline and end of intervention using a paired t-test or Wilcoxon-signed rank.

All 20 participants with Crohn's disease completed the feasibility study. Mean change in frequency of emulsifier intake during the trial was $-94.6 \%$ (SD 10.27\%). Most participants (90\%) found the diet more difficult to follow, but equally appetising (95\%), compared to habitual baseline diet. There was a significant improvement in Crohn's disease-related symptom scores $(3.0 \mathrm{vs} 1.4, \mathrm{p}=0.006)$, perceived disease-control score $(13.5$ vs $15.5, \mathrm{p}=0.026)$ and FR-QoL score $(81.5$ vs 90.0, $\mathrm{p}=0.028)$, between baseline and the end of the low emulsifier diet. Compared with baseline habitual diet, the low emulsifier diet resulted in statistically significant reductions in intakes of energy ( 2055 vs $1855 \mathrm{kcal}, \mathrm{p}=0.025)$, carbohydrate $(187 \mathrm{vs} 183 \mathrm{~g}, \mathrm{p}=0.048)$, saturated fat $(31 \mathrm{vs} 25 \mathrm{~g}$, $\mathrm{p}=0.048)$, sodium ( 2465 vs $1991 \mathrm{mg}, \mathrm{p}=0.028$ ), calcium ( $813 \mathrm{vs} 744 \mathrm{mg}, \mathrm{p}=0.015)$, niacin (36.7 vs $33.0 \mathrm{mg}, \mathrm{p}=0.021)$, and vitamin $\mathrm{B}_{12}(4.98 \mathrm{vs} 4.09 \mu \mathrm{g}$, $\mathrm{p}=0.021)$. Nutrient changes were not clinically significant as the proportion of participants meeting national recommended requirements was not significantly different. There were no changes in weight, body mass index or fat-free mass between baseline and end of trial.

Table 1: Diet feasibility and acceptability responses at end of the feasibility study

\begin{tabular}{|c|c|c|c|c|}
\hline \multirow[t]{2}{*}{ Question } & \multicolumn{2}{|r|}{ Response } & \multicolumn{2}{|l|}{$\mathrm{n}(\%)$} \\
\hline & No & Slightly more & More & Much more \\
\hline Meal preparation was more difficult & $11(55)$ & $6(30)$ & $3(15)$ & $0(0)$ \\
\hline Longer time spent preparing and cooking meals & $7(35)$ & $11(55)$ & $2(10)$ & $0(0)$ \\
\hline Longer time spent food shopping & $0(0)$ & $8(40)$ & $8(40)$ & $3(15)$ \\
\hline Finding suitable foods when shopping was more difficult & $2(10)$ & $11(55)$ & $6(30)$ & $1(5)$ \\
\hline Finding suitable foods when eating out was more difficult & $6(30)$ & $1(5)$ & $3(15)$ & $10(50)$ \\
\hline The flavour of meals and snacks was less appetising & $19(95)$ & $1(5)$ & $0(0)$ & $0(0)$ \\
\hline More money spent on food shopping and eating out & $5(25)$ & $8(40)$ & $2(10)$ & $0(0)$ \\
\hline The diet was more difficult & $2(10)$ & $14(70)$ & $4(20)$ & $0(0)$ \\
\hline
\end{tabular}

A diet low in emulsifiers was feasible and safe in people with Crohn's disease when delivered by a dietitian. Most participants adhered to the low emulsifier diet and found it as appetising. Crohn's disease-related symptoms and perceived disease-control improved, but could reflect a placebo response, which cannot be assessed without a control group. Investigation of the clinical efficacy of low emulsifier diet in Crohn's disease in an adequately powered clinical trial is warranted.

1. Chassaing B et al. (2015). Nature 519 (7541), 92-6. 